\title{
COST-TIME TRADE-OFF IN THREE-AXIAL SUMS' TRANSPORTATION PROBLEM
}

\author{
LAKSHMISREE BANDOPADHYAYA ${ }^{1}$
}

(Received 18 September 1990; revised 18 December 1992)

\begin{abstract}
The bi-objective Cost-time Trade-off Three Axial Sums' Transportation Problem is shown to be equivalent to a single-objective standard Three Axial Sums' problem, which can be solved easily by the existing efficient methods. The equivalence is established for some specially defined solutions termed as Lexicographic optimal solutions with minimum pipe-line.
\end{abstract}

\section{Introduction}

In 1961, Charnes and Cooper [3] discussed an approach to the solutions of managerial level problems involving multiple conflicting objectives (or goals). In 1962, Ignizio [8] was the first person to study the application of goalprogramming to an engineering design problem. While working on the Saturn/Apollo antenna designing program (the U.S. moon-landing mission), which had to satisfy a number of conflicting objectives, he extended the original goal programming concept of Charnes and Cooper to a non-linear model. Later, Ignizio $[9,10]$ developed further extensions of the goal programming approach. In 1965, Y. Iziri [11] proposed the inclusion of the concept of "preemptive priorities". He suggested that a priority be given to each objective or a set of commensurable objectives in the problem. In practice, this concept is achieved through finding the lexicographic minimum of an ordered vector. The Three Axial Sums' Problem was first defined by E. D. Schell [13] in 1955. An efficient solution method was suggested by A. Corban [4] in 1964. The problem of

${ }^{1}$ Deshbandhu College, University of Delhi.

(C) Australian Mathematical Society, 1994, Serial-fee code 0334-2700/94 
minimising the duration of transportation has been studied by many authors like Hammer [6, 7], Garfinkel and Rao [5], Szwarc [16], Bhatia, Swaroop and Puri [1, 2], Sharma and Swaroop [15], Seshan and Tikekar [14] and Prakash [12]. Some of these authors have also tried to unify the two problems by giving high and low priorities to the two objectives. This is what has come to be known as the cost-time trade-off problem. In the present paper cost-time trade-off has been studied in a bi-objective Three-Axial Sums' Transportation Problem. It is established that such Trade-off Three-index Problems are equivalent to a single objective standard Three-Axial Sums' Problem. The reduction to a single objective form has been achieved by assigning suitable weights to the objectives. The weights have been clearly defined to give high and low priorities to cost and time respectively. The equivalence is established for some specially defined solutions termed as lexicographic optimal solutions with minimum pipeline.

\section{Theoretical development}

A cost-time trade-off Three Axial Sums' problem is:

$$
P(1):\left\{\begin{aligned}
\text { Minimize } & C=\sum_{I} \sum_{J} \sum_{K} c_{i j k} x_{i j k} \\
\text { subject to } & \sum_{J}^{T} \sum_{K} x_{i j k}=a_{i}, \quad i \in I \\
& \sum_{I} \sum_{i j k}^{K} x_{i j k}=b_{j}, \quad j \in J \\
\sum_{I} \sum_{J} x_{i j k}=c_{k}, \quad k \in K & \geq 0,
\end{aligned}\right.
$$

where $I, J, K$ are respectively the index-sets for the warehouses, markets and the modes of transport.

$a_{i} \quad$ is the availability at the $i^{t h}$ warehouse,

$b_{j} \quad$ is the demand at the $j^{\text {th }}$ market,

$c_{k} \quad$ is the capacity of the $k^{t h}$ mode of transport,

$t_{i j k}$ is the total time taken by the $k^{t h}$ mode in transporting from $i^{\text {th }}$ warehouse to $j^{\text {th }}$ market.

$c_{i j k}$ is the per unit cost of transporting from $i^{\text {th }}$ warehouse to $j^{t h}$ market by the $k^{\text {th }}$ mode.

$x_{i j k}$ is the total amount transported from $i^{\text {th }}$ warehouse to $j^{\text {th }}$ market by the $k^{t h}$ mode. 
The method of solving $P(1)$ is to optimise the cost minimisation transportation problem in $P(1)$ by any of the methods suggested by Schell, [13], or Corban [4]. Then locating all the alternate optimal solutions corresponding to the cost, choose the one with the minimum time. The corresponding solution that is obtained is the optimal solution of $P(1)$ with cost as the first priority and the time as second priority.

The triplet $(X, C, T)$ will be used to denote the optimal solution $X=\left\{x_{i j k}\right\}$ of $P(1)$ with cost as first priority and time as second, where $C$ is the optimal cost and $T$ is the corresponding minimum time.

It will be shown that the problem $P(1)$ with cost as first priority and time as second can be reduced to the following single objective Three-Axial Sums' problem.

$$
P(2):\left\{\begin{aligned}
\text { Minimize } & Z=M_{0} \sum_{I} \sum_{J} \sum_{K} c_{i j k} x_{i j k}+\sum_{s=1}^{q}\left(M_{s} \sum_{k_{s}} x_{i j k}\right) \\
\text { subject to } & \sum_{J} \sum_{K} x_{i j k}=a_{i}, \\
\sum_{I}^{K} \sum_{K}^{K} x_{i j k} & =b_{j}, \\
\sum_{I} \sum_{J}^{K} x_{i j k} & =c_{k}, \\
x_{i j k} & \geq 0,
\end{aligned}\right.
$$

where $L_{1}, L_{2}, \ldots, L_{q}$ and $M_{0}, M_{1}, \ldots, M_{q}$ are defined below:

Let

$$
\max _{l, J, K} t_{i j k}=\beta_{1}, \quad \min _{l, J, K} t_{i j k}=\beta_{q} .
$$

The set $\left\{t_{i j k}: i \in I, j \in J, k \in K\right\}$ is sub-divided into subsets $L_{1}, L_{2}, \ldots, L_{q}$ satisfying the following two conditions:

(i) $L_{u}$ contains the $t_{i j k}$ 's having the same numerical value, $u=1,2, \ldots, q$;

(ii) $\beta_{1}>\beta_{2}>\ldots>\beta_{q}$.

$M_{0}, M_{1}, \ldots, M_{q}$ are the priority factors associated with $\sum_{I} \sum_{J} \sum_{K} c_{i j k} x_{i j k}$, $\sum_{L_{1}} x_{i j k}, \sum_{L_{2}} x_{i j k}, \ldots, \sum_{L_{q}} x_{i j k}$ respectively, $\sum_{L_{s}} x_{i j k}$ denoting the sum of all those $x_{i j k}$ 's with corresponding $t_{i j k} \in L_{s} . M_{0}, M_{1}, \ldots, M_{q}$ are all positive and they are so defined, that the sign of $\sum_{k=0}^{q} \alpha_{k} M_{k}$ is the same as the non-zero $\alpha_{k}$, with smallest subscript $k$ in it, irrespective of the other $\alpha_{k}$ 's.

DEFINITION. LEXICOGRAPHICALLY OPTIMAL SOLUTION WITH MINIMUM PIPELINE An optimal solution $\left(X^{*}, C^{*}, T^{*}\right)$ of the cost-time Trade-off Three Axial Sums' 
Transportation problem $P(1)$, will be called lexicographically optimal with minimum pipeline, if for any solution $\left(X, C^{*}, T^{*}\right)$, either

$$
\sum_{L_{s}} x_{i j k}^{*}<\sum_{L_{s}} x_{i j k}
$$

where $T^{*} \in L_{s}, X^{*}=\left\{x_{i j k}^{*}\right\}, X=\left\{x_{i j k}\right\}$, or if

$$
\sum_{L_{s}} x_{i j k}^{*}=\sum_{L_{s}} x_{i j k}
$$

and $T_{1}^{*}$ is the second largest time for both the solution $X$ and $X^{*}$, so that $T_{1}^{*} \in L_{s+1}$, then

$$
\sum_{L_{s+1}} x_{i j k}^{*}<\sum_{L_{s+1}} x_{i j k}
$$

The equivalence of the problem $P(1)$ with cost as first priority and the time as second with the problem $P(2)$ is established, through the following theorem and its converse.

THEOREM. If $\left(X^{*}=\left\{x_{i j k}^{*}\right\}_{I \times J \times K}, C^{*}, T^{*}\right)$ is a lexicographicallyoptimal solution of $P(1)$ with minimum pipeline, then it is also optimal for $P(2)$.

Proof. Obviously $X^{*}$ is feasible for $P(2)$, as the feasibility conditions of $P(1)$ and $P(2)$ are same.

Now we show $X^{*}$ is optimal for $P(2)$.

Let $X=\left\{x_{i j k}\right\}_{l \times J \times K}$ be any feasible solution of $P(2)$ with corresponding cost as $C$ and time $T$.

Now $C \geq C^{*}$, as $X$ is also feasible for $P(1)$.

CASE (I) $C>C^{*}$.

That is,

$$
\sum_{I} \sum_{J} \sum_{K} c_{i j k} x_{i j k}>\sum_{I} \sum_{J} \sum_{K} c_{i j k} x_{i j k}^{*}
$$

which implies

$$
\sum_{I} \sum_{J} \sum_{K} c_{i j k}\left(x_{i j k}-x_{i j k}^{*}\right)>0
$$

Now

$$
\begin{aligned}
Z-Z^{*} & =M_{0} \sum_{l} \sum_{J} \sum_{K} c_{i j k}\left(x_{i j k}-x_{i j k}^{*}\right)+\sum_{u=1}^{q} M_{u} \sum_{L_{u}}\left(x_{i j k}-x_{i j k}^{*}\right) \\
& =\sum_{u=0}^{q} M_{u} \alpha_{u} .
\end{aligned}
$$


Since $\alpha_{0}>0$ (using (1)),

$$
\begin{aligned}
Z- & \left.Z^{*}>0 \text { (by definition of } M_{0}, M_{1} \ldots, M_{q}\right) \\
& \Rightarrow Z>Z^{*} \\
& \Rightarrow X^{*} \text { has the minimum objective function value compared to } \\
& \quad \text { any feasible solution of } P(2) . \\
& \Rightarrow X^{*} \text { is optimal for } P(2) .
\end{aligned}
$$

CASE (II) $C=C^{*}$

This shows that $X^{*}, X$ are two alternate optimal solutions of $P(1)$ with respect to cost minimisation.

The optimality of $\left(X^{*}, C^{*}, T^{*}\right)$ suggests that $T^{*}$ is the minimum of all the times corresponding to the alternate optimal solutions with respect to $\operatorname{cost} C^{*}$.

Thus $T^{*} \leq T$.

In the case $T^{*}<T, T^{*} \in L_{s}$ and $T \in L_{p}$ then $s>p$ and

$$
\begin{array}{ll}
L_{1}=L_{2}=\ldots=L_{s-1}=\phi & \text { for } X^{*}=\left\{x_{i j k}^{*}\right\} \\
L_{1}=L_{2}=\ldots=L_{p-1}=\phi & \text { for } X=\left\{x_{i j k}\right\}
\end{array}
$$

Now $Z-Z^{*}=\sum_{k=1}^{q} M_{k} \sum_{L_{k}}\left(x_{i j k}-x_{i j k}^{*}\right)\left(\right.$ as $\left.C=C^{*}\right)$ has the sign of $\sum_{L_{p}}\left(x_{i j k}-\right.$ $\left.x_{i j k}^{*}\right)=\sum_{L_{p}} x_{i j k}$.

Hence

$$
\begin{aligned}
Z-Z^{*}>0 \\
\quad \Rightarrow Z>Z^{*} \\
\quad \Rightarrow X^{*} \text { is optimal for } P(2) .
\end{aligned}
$$

Again in the case $T^{*}=T$, we have $x=\left\{x_{i, k}\right\}$ as a feasible solution of $P(1)$ with the same cost $C^{*}$ and same time $T^{*}$ as the optimal solution $X^{*}$.

The definition of lexicographic optimality suggests that either (i) or (ii) holds. (i) $\sum_{L_{s}} x_{i j k}^{*}<\sum_{L_{s}} x_{i j k}$, in which case, $\sum_{L_{s}}\left(x_{i j k}-x_{i j k}^{*}\right)>0$.

Now since $L_{1}=L_{2}=\ldots=L_{s-1}=\phi$ for $X^{*}$ and $L_{1}=L_{2}=\ldots L_{s-1}=\phi$ for $X$,

$$
\begin{aligned}
Z-Z^{*} & =\sum_{u=1}^{q} M_{k} \sum_{L_{u}}\left(x_{i j k}-x_{i j k}^{*}\right) \\
& =M_{s} \sum_{L_{s}}\left(x_{i j k}-x_{i j k}^{*}\right)+\ldots+M_{q} \sum_{L_{q}}\left(x_{i j k}-x_{i j k}^{*}\right) .
\end{aligned}
$$


Hence $Z-Z^{*}$ has the sign of $\sum_{L_{s}}\left(x_{i j k}-x_{i j k}^{*}\right)>0$, which implies $Z>Z^{*}$, and hence $X^{*}$ is optimal for $P(2)$.

(ii) $\quad \sum_{L_{s}} x_{i j k}^{*}=\sum_{L_{s}} x_{i j k}$. In this case, if the second largest time for $X^{*}$ and $X$ are same, say $T_{1}$, then $T_{1} \in L_{s+1}$ and $\sum_{L_{s}+1} x_{i j k}^{*}<\sum_{L_{s}+1} x_{i j k}$ and

$$
\begin{aligned}
Z-Z^{*} & =\sum_{k=1}^{q} M_{k} \sum_{L_{k}}\left(x_{i j k}-x_{i j k}^{*}\right) \\
& =M_{s+1} \sum_{L_{s}+1}\left(x_{i j k}-x_{i j k}^{*}\right)+\ldots+M_{q} \sum_{L_{q}}\left(x_{i j k}-x_{i j k}^{*}\right)
\end{aligned}
$$

has the sign of $\sum_{L_{s}+1}\left(x_{i j k}-x_{i j k}^{*}\right)>0$. Therefore $Z-Z^{*}>0$. Hence $X^{*}$ is optimal for $P(2)$. Again if the second largest times of $X^{*}$ and $X$ are different, say $T_{1}$ and $T_{2}$ respectively, with $T_{1}<T_{2}$, and if $T_{1} \in L_{m}$ and $T_{2} \in L_{n}$ then $m>n$,

$$
\begin{aligned}
Z-Z^{*} & =\sum_{u=1}^{q} M_{u} \sum_{L_{u}}\left(x_{i j k}-x_{i j k}^{*}\right) \\
& =M_{n} \sum_{L_{n}}\left(x_{i j k}-x_{i j k}^{*}\right)+\ldots M_{q} \sum_{L_{q}}\left(x_{i j k}-x_{i j k}^{*}\right) .
\end{aligned}
$$

Since $L_{1}=L_{2} \ldots=L_{m-1}=\phi$ for $X^{*} L_{1}=L_{2} \ldots=L_{n-1}=\phi$ for $X, Z-Z^{*}$ has the sign of $\sum_{L_{n}}\left(x_{i j k}-x_{i j k}^{*}\right)=\sum_{L_{n}}\left(x_{i j k}\right)$, that is $Z-Z^{*}>0$ and so $X^{*}$ is optimal for $P(2)$.

So in all the cases with various subcases it has been proved that $\left(X^{*}, C^{*}, T^{*}\right)$ is optimal for $P(2)$.

THEOREM. (Converse): If $X^{*}=\left\{x_{i j k}^{*}\right\}$ is an optimal solution of $P(2)$ with $\sum_{I} \sum_{J} \sum_{K} c_{i j k} x_{i j k}^{*}=C^{*}$ and $T^{*}$ is the corresponding time, then $\left(X^{*}, C^{*}, T^{*}\right)$ is a lexicographically optimal solution of $P(1)$ with minimum pipeline.

Proof. Obviously $\left(X^{*}, C^{*}, T^{*}\right)$ is feasible for $P(1)$. Suppose $X \neq X^{*}$ is an optimal solution for $P(1)$ with costs $C$ and time $T$. Then $C \leq C^{*}$ implies

$$
\sum_{I} \sum_{J} \sum_{K} c_{i j k} x_{i j k} \leq \sum_{I} \sum_{J} \sum_{K} c_{i j k} x_{i j k}^{*} .
$$

It is claimed that $C=C^{*}$; for if $C<C^{*}$, then

$$
Z-Z^{*}=M_{0} \sum_{I} \sum_{J} \sum_{K} c_{i j k}\left(x_{i j k}-x_{i j k}^{*}\right)+\sum_{u=1}^{q} M_{u} \sum_{L_{u}}\left(x_{i j k}-x_{i j k}^{*}\right)
$$


(by the definition of priority factors $M_{0}, M_{1} \ldots, M_{q}$ ) has the sign of $\sum_{I} \sum_{J} \sum_{K}$ $c_{i j k}\left(x_{i j k}-x_{i j k}^{*}\right)$ which is less than zero (as $C<C^{*}$ ). But $Z-Z^{*}<0$ implies $Z<Z^{*}$ which contradicts the optimality of $X^{*}$ for $P(2)$. Thus any optimal solution of $P(1)$ must have its optimal cost as $C^{*}$.

Now, if $\left(X^{0}, C^{*}, T^{0}\right)$ is any alternate optimal solution of $P(1)$, then $T^{0} \leq T^{*}$. In the case $T^{0}<T^{*}$ and $T^{0} \in L_{s}, T^{*} \in L_{p}$ then $s>p$ and $Z^{0}-Z^{*}=$ $\sum_{u=1}^{q} M_{u} \sum_{L_{u}}\left(x_{i j k}^{0}-x_{i j k}^{*}\right)$ has the sign of

$$
\sum_{L_{p}}\left(x_{i j k}^{0}-x_{i j k}^{*}\right)=-\sum_{L_{p}}\left(x_{i j k}^{*}\right)
$$

[as $L_{1}=L_{2}=\ldots=L_{p-1}=\phi$ for $X^{*}$ and $L_{1}=L_{2}=\ldots=L_{p-1}=\ldots=$ $L_{s-1}=\phi$ for $\left.X\right]$.

Hence $Z^{0}-Z^{*}<0$, which contradicts the optimality of $X^{*}$. Therefore $T^{0}=T^{*}$. Thus any optimal solution must have optimal cost $C^{*}$ and time $T^{*}$. This implies $X^{*}$ is optimal for $P(1)$.

Again $X^{*}$ is lexicographically optimal with minimum pipeline, as any solution $\left(X, C^{*}, T^{*}\right)$ of $P(1)$ must satisfy $\sum_{L_{s}} x_{i j k}^{*} \leq \sum_{L_{s}} x_{i j k}$, for otherwise $\sum_{L_{s}} x_{i j k}^{*}>$ $\sum_{L_{s}} x_{i j k}$ will contradict the optimality of $X^{*}$.

Such a work finds its application in all problems of routing finished goods from factories to retailers using different modes of transport. A factory owner is generally satisfied when his goods are delivered at a minimum cost. The minimisation of time of delivery is his second concern. The present model will be most useful in all such cases.

\section{Acknowledgements}

The author is grateful to Dr. M. C. Puri, Associate Professor, IIT, Delhi for his kind guidance and valuable suggestions.

\section{References}

[1] H. L. Bhatia, K. Swarup and M. C. Puri, "Time-cost trade-off in a transportation problem", Opsearch 13 (1976) 129-142.

[2] H. L. Bhatia, K. Swarup and M. C. Puri, "A procedure for the time minimizing transportation problem”, Indian J. Pure Appl. Math. 8 (1977) 920-929. 
[3] A. Charnes and W. W. Cooper, Management models and industrial applications of linear programming, Vols 1 and 2 (Wiley, New York, 1961).

[4] Adrian Corban, "A multi-dimensional transportation problem", Rev. Roum. Math. Pure and Appl. 9 (8) (1964) 721-735.

[5] R. S. Garfinkel and M. R. Rao, "The bottleneck transportation problem", Nav. Res. Log. Quart. 18 (1971) 465-472.

[6] P. L. Hammer, “Time minimizing transportation problems", Nav. Res. Log. Quart. 16 (1969) 345-357.

[7] P. L. Hammer, “Time minimizing transportation problems", Nav. Res. Log. Quart. 18 (1971) $487-490$.

[8] J. P. Ignizio, "S-II Trajectory Study and Optimum Antenna Placement", North American Aviation Report S1D-63, Downey, California, 1963.

[9] J. P. Ignizio, Goal programming and extensions (Heath (Lexington Books), Lexington, Mass, 1976).

[10] J. P. Ignizio, "Goal programming: A tool for multi-objective analysis", J. Oper. Res. Soc. 29 (II) (1978) 1109-1119.

[11] Y. lziri, Management goals and accounting for control (Rand Mcnally, Chicago, 1965).

[12] S. Prakash, "On minimizing the duration of transportation", Proc. Ind. Acad. Sci. (Math. Sci) 91 (1982) 53-57.

[13] E. D. Schell, "Distribution of a product by several properties", in Proceedings of the $2^{\text {nd }}$ Symposium in Linear programming, DCS/Comptroller H.Q.U.S.A.F., Washington D. C. (1955).

[14] C. R. Seshan and V. G. Tikekar, "On Sharma-Swarup algorithm for time minimizing transportation problems", Proc. Ind. Acad. Sci. (Math. Sci) 89 (1980) 101-102.

[15] J. K. Sharma and K. Swarup, "Time minimizing transportation problem", Proc. Ind. Acad. Sci. (Math. Sci) 86 (1977) 513-518.

[16] W. Szwarc, "Some remarks on the time minimizing transportation problem", Nav. Res. Log. Quart. 18 (1971) 473-485. 